\title{
The Effect of Individualized Versus Standardized Parenteral Nutrition on Body Weight in Very Preterm Infants
}

\author{
Vincent H. M. Evering ${ }^{\mathrm{a}}$, Peter Andriessen ${ }^{\mathrm{b}, \mathrm{c}}$, Carola E. P. M. Duijsters ${ }^{\mathrm{b}}$, \\ Jeroen Brogtrop ${ }^{\text {a }}$, Luc J. J. Derijks ${ }^{\text {a, d }}$
}

\begin{abstract}
Background: This study was designed to evaluate whether standardizing total parenteral nutrition (TPN) is at least non-inferior to TPN with individualized composition in premature infants with a gestational age $(\mathrm{GA})<32$ weeks.

Methods: In this retrospective cohort study, all preterm born in or transferred to Maxima Medical Center (MMC) within 24 hours after birth with a GA $<32$ weeks were included. The individualized group (2011) was compared to the partially standardized group (2012) and completely standardized group (2014) consequently. The primary endpoint was difference in growth. Secondary endpoints included differences in electrolyte concentrations.

Results: A total of 299 preterm were included in this study. When comparing weight gain, the infants in the (partially) standardized group demonstrated significantly $(\mathrm{P}<0.05)$ less weight loss during the first days of life and grew faster subsequently in the following days than the individualized TPN regimen. Furthermore, significant differences in abnormal serum sodium, chloride, calcium, creatinine, magnesium and triglycerides values were demonstrated.
\end{abstract}

Conclusion: TPN with a (partially) standardized composition revealed to be at least non-inferior to TPN with an individualized composition.

Keywords: Parenteral nutrition; Preterm infant; Weight gain

\section{Introduction}

Very preterm infants in a neonatal intensive care unit (NICU)

\footnotetext{
Manuscript accepted for publication January 12, 2017

aDepartment of Clinical Pharmacy, Maxima Medical Center, Veldhoven, The Netherlands

bDepartment of Pediatrics, Maxima Medical Center, Veldhoven, The Netherlands

${ }^{\circ}$ Faculty of Health, Medicine and Life Science, Maastricht University, Maastricht, The Netherlands

${ }^{\mathrm{d} C o r r e s p o n d i n g ~ A u t h o r: ~ L u c ~ J . ~ J . ~ D e r i j k s, ~ D e p a r t m e n t ~ o f ~ C l i n i c a l ~ P h a r m a c y, ~}$ Maxima Medical Center, PO Box 7777, 5500 MB Veldhoven, The Netherlands.Email: 1.derijks@mmc.nl
}

doi: https://doi.org/10.14740/jocmr2893w are completely dependent on total parenteral nutrition (TPN) for adequate food intake until enteral feeding in sufficient amounts is well tolerated. For now, there is no consensus whether individualized formulations are superior to standardized formulations in achieving TPN goals [1]. Besides formulation, an adequate nutrition protocol, especially during the first days of life, is of great importance [2.3].

Before 2012, it was common practice in Maxima Medical Center (MMC) to feed the very preterm with a pharmacy compounded, all-in-one TPN with individualized composition within $24 \mathrm{~h}$ after birth. In March 2012, MMC switched to an all-in-one TPN with a partially standardized composition (Numeta ${ }^{\circledR}$ G13\%E, Baxter B.V., Utrecht, The Netherlands) and in December 2013 to an all-in-one TPN with a completely standardized composition (NEOmix) consequently.

Our assumption was that standardized TPN is at least comparable to individualized TPN and superior in respect of reducing compounding errors, prescribing errors and infection risk due to a less complex aseptic preparation. Furthermore, we hypothesized standardized TPN could result in a cost reduction. A presumed disadvantage of standardized TPN was less biochemical control [1, 4-6].

We therefore conducted a study to demonstrate whether standardizing TPN is at least non-inferior to TPN with individualized composition in premature infants with a gestational age $(\mathrm{GA})<32$ weeks.

\section{Methods}

\section{Subjects}

The Institutional Review Board/Independent Ethics Committee (IRB/IEC) of MMC declared that this study did not have to be reviewed by a medical ethics board according to Dutch Law on Medical Research with Humans (WMO) as it concerned a retrospective cohort study. In total 299 infants with a GA $<32$ weeks were included. Study data were collected in the same period of the year (April - September) to prevent seasonal influences. Premature infants who died within $<72 \mathrm{~h}$ were excluded because mortality was not assumed to be associated with TPN.

\section{TPN}

The 2011 group received an individualized TPN (ITPN, $\mathrm{n}=$ 
Table 1. Composition of TPN

\begin{tabular}{llll}
\hline Composition per $\mathbf{1 0 0} \mathbf{~ m L}$ & ITPN: BAXA compounder & PSTPN: Numeta G13\%E 3C & STPN: NEOmix \\
\hline Energy (kcal) & Variable & 91 & 66 \\
Osmolarity (mOsmol/L) & Variable & 1,155 & 805 \\
Protein (g) & Variable & 3.1 & 2.6 \\
Triglycerides & Variable & 2.5 & 2.0 \\
Glucose (g) & Variable & 13.3 & 8.9 \\
Sodium (mmol) & Variable & 2.2 & 2.1 \\
Potassium (mmol) & Variable & 2.1 & 0.66 \\
Magnesium (mmol) & Variable & 0.42 & 0.1 \\
Calcium (mmol) & Variable & 1.25 & 0.7 \\
Phosphate (mmol) & Variable & 1.26 & 0.93 \\
Chloride (mmol) & Variable & 3.1 & 1.39 \\
\hline
\end{tabular}

ITPN: individualized total parental nutrition; PSTPN: partially standardized total parental nutrition; STPN: standardized total parental nutrition.

94), the 2012 group received a partially standardized TPN (PSTPN, $\mathrm{n}=101$ ) and the 2014 group received a completely standardized TPN (STPN, $n=104)$. The ITPN was composed of the following components: potassium sodium phosphate 2 $\mathrm{mmol} / \mathrm{mL}$ (Addiphos ${ }^{\circledR}$, Fresenius Kabi Nederland B.V., Zeist, The Netherlands), sodium chloride $10 \%$, potassium chloride 1 $\mathrm{mmol} / \mathrm{mL}$, glucose $50 \%$, trace elements (Peditrace ${ }^{\circledR}$, Fresenius Kabi Nederland B.V., Zeist, The Netherlands), calcium glubionate $137.5 \mathrm{mg} / \mathrm{mL}$ (Calcium-Sandoz ${ }^{\circledR}$, Sandoz B.V., Almere, The Netherlands), magnesium chloride, vitamines (Vaminol$\operatorname{act}^{\circledR}$, Fresenius Kabi Nederland B.V., Zeist, The Netherlands), lipid emulsion (ClinOleic $^{\circledR}$ 20\%, Baxter B.V., Utrecht, The Netherlands), and water for injections (WFI). Partially standardized in PSTPN means a fixed dose of vitamins (Vitintra Infant ${ }^{\circledR}+$ Soluvit $\mathrm{N}^{\circledR}$, both Fresenius Kabi Nederland B.V., Zeist, The Netherlands) and trace elements (Peditrace ${ }^{\circledR}$, Fresenius Kabi Nederland B.V., Zeist, The Netherlands), a variable amount of WFI and/or potassium was added to the TPN bag.
Moreover, if lipids were not required, the bag design allows activation of peel seals between amino acids/electrolytes and glucose chambers only. Consequently, the fixed dose of lipidsoluble vitamins was also not added to the bag. Table 1 shows the TPN composition.

Furthermore, because of new insights adapted from international guidelines, a new national nutrition protocol was introduced in 2012 [2]. Before 2012, amino acids and lipids were increased stepwise [7], while after introduction of the new national protocol infants received high maintenance doses from the very beginning. The nutrition protocols are presented in Table 2.

Outcome measures were mean cumulative weight gain and mean daily weight gain velocity. In addition, TPN-associated biochemical values (electrolytes and triglycerides) were studied.

In this non-inferiority designed study, comparable weight gain $(25 \mathrm{~g} / \mathrm{kg} /$ day $)$ was assumed. A total sample size of 192

Table 2. Nutrition Protocol for Infants With a Gestational Age $(G A)<32$ Weeks

\begin{tabular}{|c|c|c|}
\hline & ITPN & PSTPN and STPN \\
\hline Glucose (g/kg/day) & $\begin{array}{l}\text { Day 0: } 8-14 \\
\text { From day 1: } \max 18\end{array}$ & $\begin{array}{l}\text { Day 0: } 6-7 \\
\text { From day 1: } \max 17\end{array}$ \\
\hline Lipids (g/kg/day) & Day 0: 0 , stepwise each day plus $0.5 \mathrm{~g} / \mathrm{kg} / \mathrm{day}$ to $\max 3.5$ at day 6 & $\begin{array}{l}\text { Day 0: } 0 \\
\text { From day } 1: \max 3.5\end{array}$ \\
\hline Fluid intake (equal in all groups) & \multicolumn{2}{|l|}{$\begin{array}{l}\text { Day 0: } 60-80 \mathrm{~mL} / \mathrm{kg} \text { (depending on birth weight), stepwise } \\
\text { increase } 20 \mathrm{~mL} / \mathrm{kg} \text { to } \max 160-180 \mathrm{~mL} / \mathrm{kg} / \text { day }\end{array}$} \\
\hline Enteral intake (equal in all groups) & \multicolumn{2}{|c|}{$\begin{array}{l}\text { Days } 1 \text { - 3: start minimal enteral feeding (MEF) } 6-12 \times 0.5 \mathrm{~mL} \text { breastmilk or Nenatal }{ }^{\circledR} \\
\text { (depending on birth weight) } \\
\text { From day } 4 \text { : stepwise increase } 20 \mathrm{~mL} / \mathrm{kg} / \text { day, max } 160-180 \mathrm{~mL} / \mathrm{kg} / \text { day } \\
\text { If enteral feeding } \geq 120 \mathrm{~mL} / \mathrm{kg} / \text { day, TPN is substituted by glucose } 10 \%\end{array}$} \\
\hline
\end{tabular}

GA: gestational age; ITPN: individualized total parental nutrition; PSTPN: partially standardized total parental nutrition; STPN: standardized total parental nutrition. 
Table 3. Characteristics of 299 Infants $<32$ Weeks of GA Hospitalized in the NICU

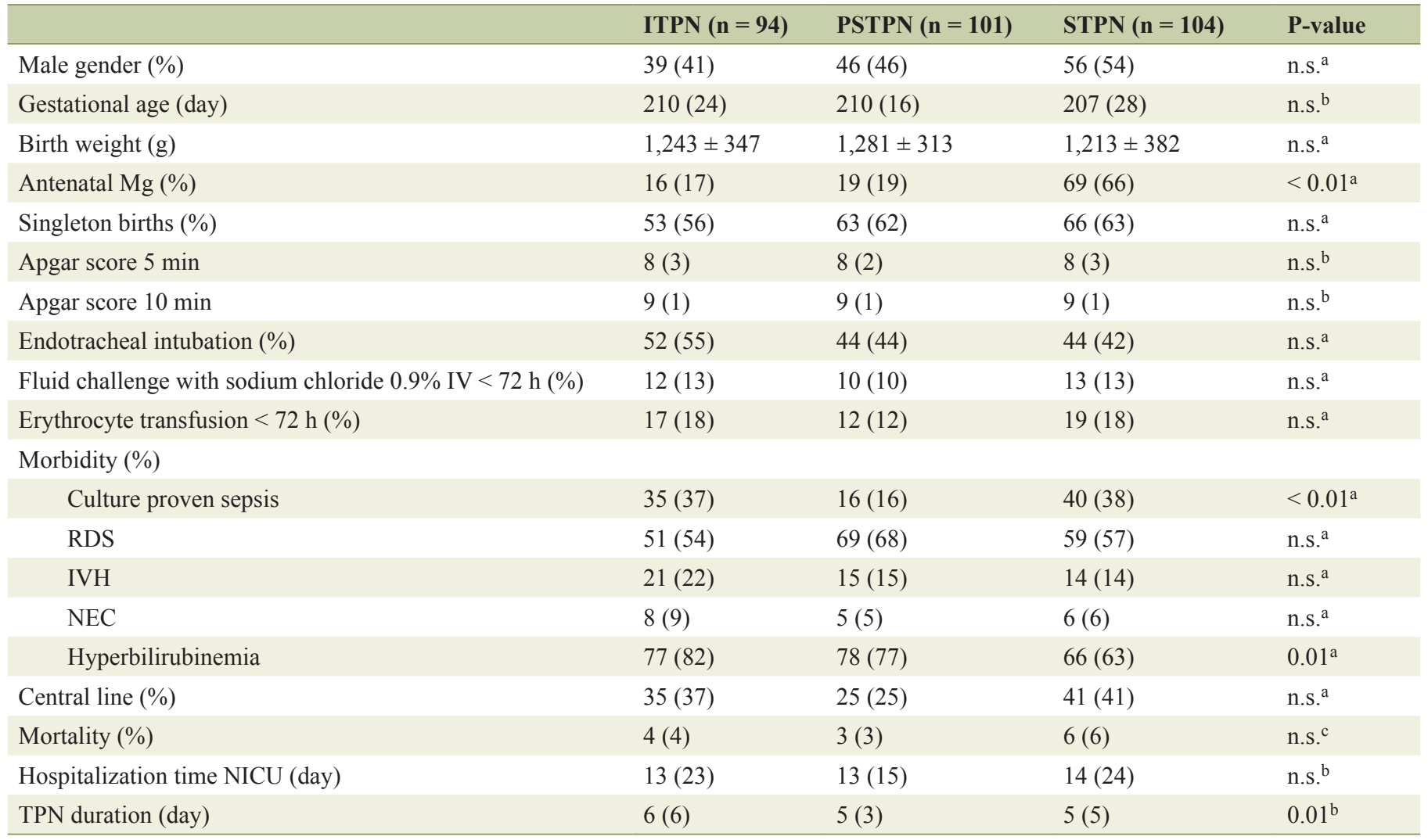

${ }^{a}$ Chi-square test. ${ }^{b}$ Kruskal-Wallis. 'Fisher's exact test. NICU: neonatal intensive care unit; GA: gestational age; ITPN: individualized total parental nutrition; PSTPN: partially standardized total parental nutrition; STPN: standardized total parental nutrition; RDS: respiratory distress syndrome; IVH: intraventricular hemorrhage; NEC: necrotizing enterocolitis; TPN: total parenteral nutrition. Normal data are expressed as mean \pm SD, otherwise median (interquartile range).

(each group 64) was calculated based on a statistical power of 0.8 and type I error rate $(\alpha)$ of 0.05 . To assure complete data, the total sample size was increased to 300 (each group 100).

Normal data were expressed as mean $\pm \mathrm{SD}$, otherwise median (interquartile range). One-way ANOVA (with Bonferroni correction or Tanane post hoc analysis) was used for testing group differences on parametric continuous data. For non-parametric continuous data, the Kruskal-Wallis and Mann-Whitney U were used. Multivariate analysis was used to study patient characteristics. Categorical data were analyzed with Chi-square or Fisher exact. Statistical analyses were performed with SPSS (version 19.0).

\section{Results}

Patient characteristics are presented in Table 3. In general, the three TPN groups were comparable. Differences between the three groups reached statistical significance for culture proven sepsis during hospitalization, antenatal $\mathrm{Mg}$ administration and TPN duration.

The mean cumulative weight gain during the first 3 weeks of life was significantly different between the groups, with the highest weight gain in the standardized group (Fig. 1). Sig- nificant differences were reached at almost all time points between ITPN and both PSTPN and STPN, respectively.

Mean daily weight gain velocity during the first 3 weeks of life was not different in general, with only significant differences on days 1 - 3, 6 - 7 and 20 (Fig. 2).

The median postnatal age of maximum weight loss was day 4 for the ITPN $(95 \%$ CI: 3.6 - 4.5) and day 3 for the PSTPN (95\% CI: 2.5 - 3.5) and STPN (95\% CI: 2.5 - 3.5), respectively. The mean maximum weight loss after birth was $-120 \mathrm{~g} / \mathrm{kg}$ in the ITPN group, $-80 \mathrm{~g} / \mathrm{kg}$ in the PSTPN group and $-75 \mathrm{~g} / \mathrm{kg}$ birth weight in the STPN group. Differences were significant between ITPN and both MSTPN and STPN, respectively. The median postnatal age of birth weight recovery was day 12 for the ITPN (95\% CI: 11.0 - 13.0), day 10 for the PSTPN (95\% CI: 9.5 - 10.5) and day 8 for the STPN (95\% CI: 7.1 - 8.9), reaching statistical significance between all groups.

Multivariate analysis of patient characteristics (Table 3) demonstrated no significant influence on primary endpoints.

In the first week of life, no differences in glucose and urea were demonstrated between the three groups. The ITPN group (26\%) revealed more hypertriglyceridemia, defined as triglycerides $>2 \mathrm{mmol} / \mathrm{L}$, compared to PSTPN $(8 \%)$ and STPN (16\%), respectively. No clinically relevant differences in electrolyte concentrations were observed between the three 


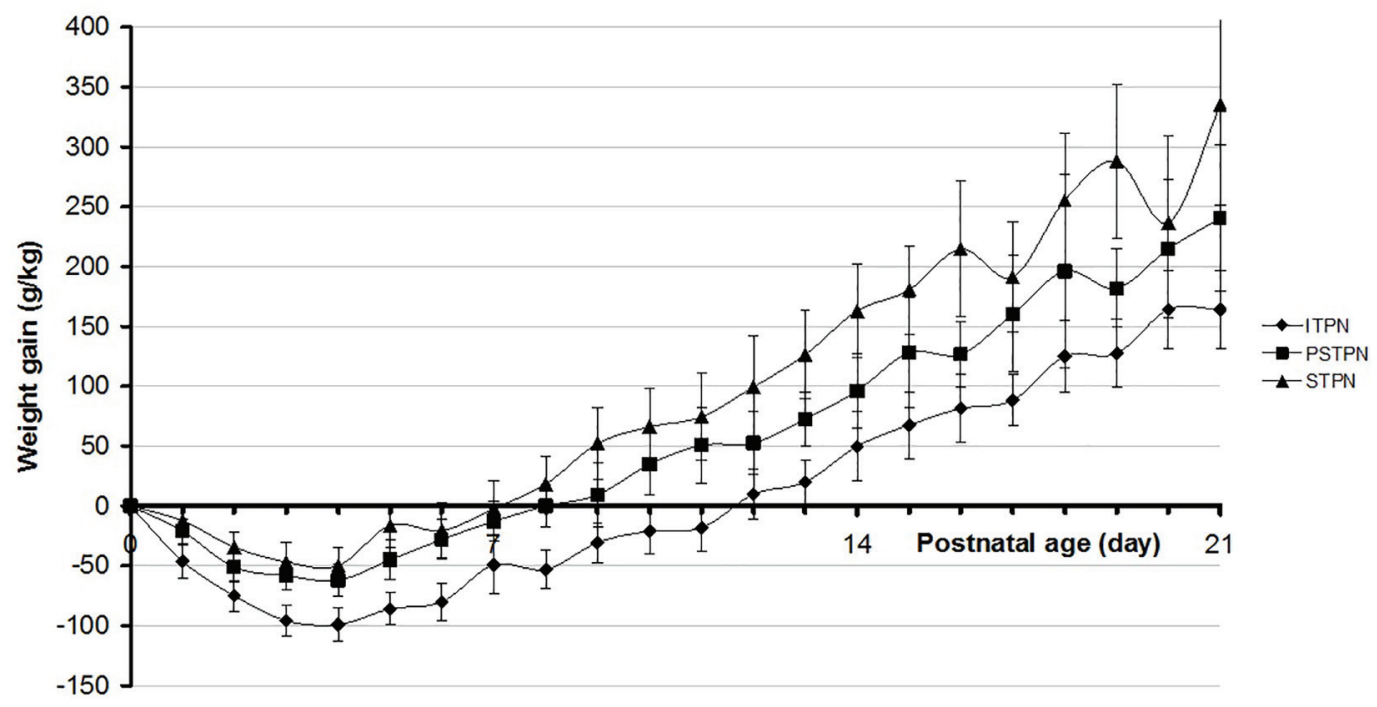

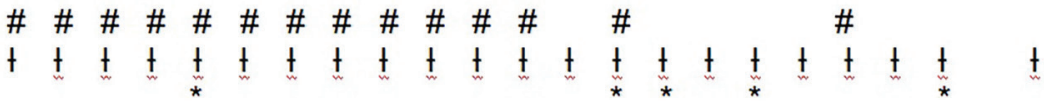

Figure 1. Mean cumulative weight gain in the first 3 weeks of life. \#Significant between ITPN versus PSTPN. ${ }^{\dagger}$ Significant between ITPN versus STPN. *Significant between PSTPN versus STPN. ITPN: individualized total parental nutrition; PSTPN: partially standardized total parental nutrition; STPN: standardized total parental nutrition.

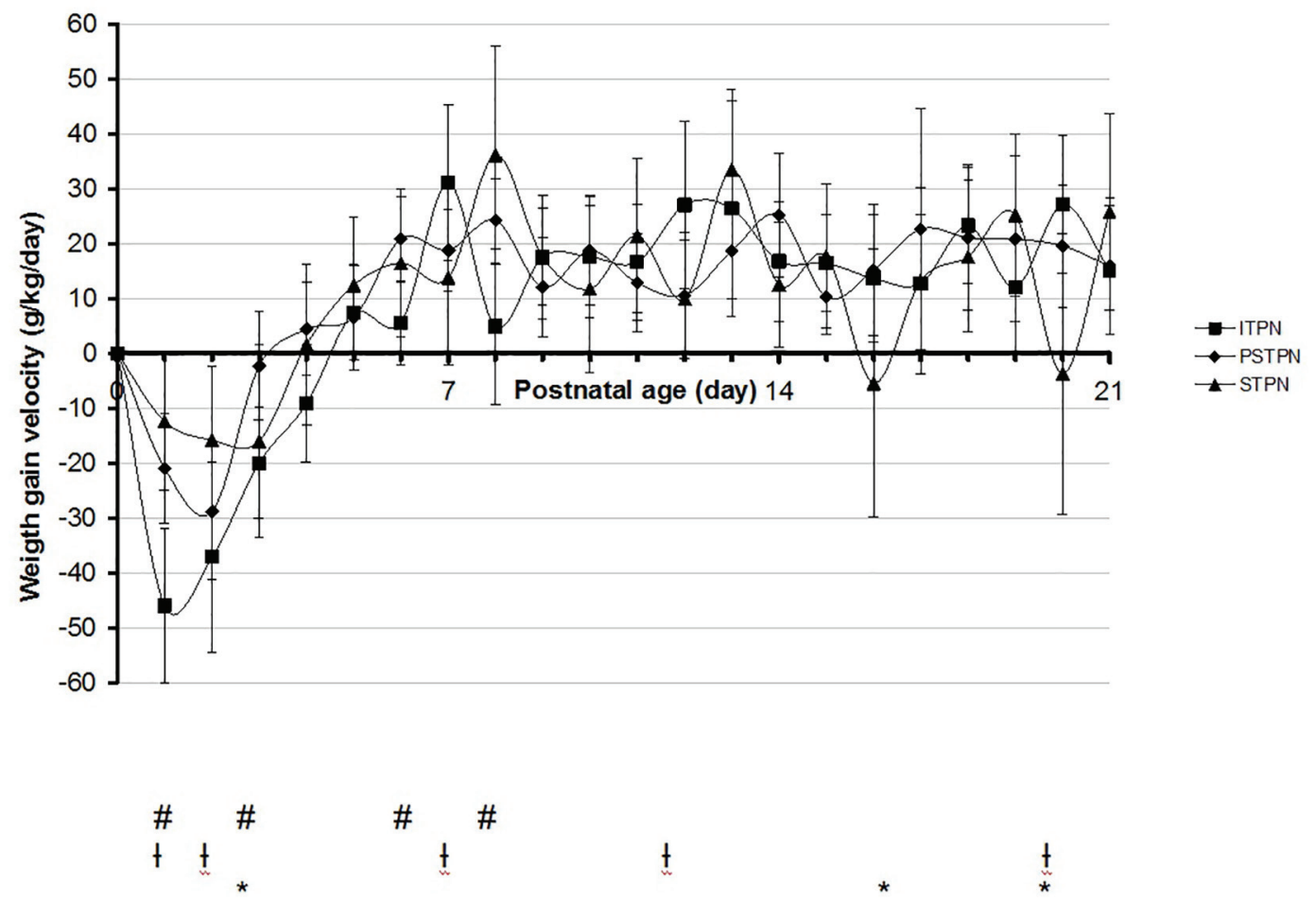

Figure 2. Mean daily weight gain velocity in the first 3 weeks of life. \#Significant between ITPN versus PSTPN. ${ }^{\dagger}$ Significant between ITPN versus STPN. *Significant between PSTPN versus STPN. ITPN: individualized total parental nutrition; PSTPN: partially standardized total parental nutrition; STPN: standardized total parental nutrition. 
groups.

\section{Discussion}

Adequate nutrition is essential for weight gain in the first days of life $[2,8]$. In this study, we demonstrated both PSTPN and STPN to be at least non-inferior to ITPN. To date, no consensus is reached what level of standardization leads to better results. One would expect to achieve better nutrition goals with ITPN, tailored to individual needs. Indeed, Smolkin et al showed more weight gain in a population with individualized TPN and attributed their finding to higher caloric intake [9]. On the other hand, Lacobelli et al described less weight loss and subsequently more weight gain in the first week of life in a comparable preterm population treated with standardized TPN [5]. They attributed their findings to a significant higher amount of amino acids and calorie intake. This may also be a plausible explanation for our finding: both PSTPN and STPN group received more amino acids during the first days of life compared to ITPN due to a change in the local nutrition protocol. Thus, it is plausible that the higher amount amino acids and caloric intake contributed to better mean daily weight gain. This assumption is supported by data from previous studies [2, $3,5,9]$. Furthermore, the duration of TPN administration was significantly shorter for both PSTPN and STPN, possibly related to an earlier switch to enteral feeding.

Also, in this study, both the postnatal age at maximum weight loss and age at birth weight recovery were lower and the duration of TPN was shorter, compared to data from previous investigations $[8,10]$. This is explained by our nutrition protocol in which TPN, in particular amino acids, is started earlier after birth which is proven to be beneficial $[5,11]$.

Remarkably, we observed a higher incidence of a culture proven sepsis in the ITPN and STPN cohorts compared to the PSTPN. These differences cannot be explained by basic patient characteristics such as GA, birth weight and postnatal age (Table 3). The higher incidence of culture proven sepsis in the ITPN group could at least in part be explained by the more complex TPN preparation compared to PSTPN. Notably, this was the main reason for switching to PSTPN. However, we cannot explain the difference in sepsis incidence between the PSTPN and STPN group.

Both standardized TPN preparations contain substantial amounts of electrolytes, added by the manufacturers [12]. Indeed, the standardized preparations resulted in higher incidences of hypernatremia and hyperchloremia and a lower incidence of hyponatremia compared to the individualized preparation. The higher incidence of hypernatremia and hyperchloremia in the standardized preparation groups cannot be explained by insensible water loss as the PSTPN and STPN groups demonstrated significantly less weight loss compared to the ITPN group [2]. The calcium concentration was lower in the STPN group compared to the other TPN preparations and calcium concentration advised in international guidelines [2, 7].

During our study, the PSTPN product was withdrawn from the market because of a reported higher risk on developing hypermagnesemia. Indeed, we observed a relatively high incidence of hypermagnesemia in the PSTPN group compared to the STPN group. However, there was no difference between the PSTPN group and the ITPN group, the latter being individualized by electrolyte levels, including magnesium. Therefore, our results do not support market withdrawal.

This retrospective study was subjected to several methodological limitations. First, daily total fluid intake, enteral intake and caloric intake were not taken into account. The local nutrition protocols for the ITPN, PSTPN and STPN group were similar regarding targets for total fluid intake and enteral intake. However, the influence of these parameters on weight gain as possible confounders cannot be ruled out. Second, as mentioned before, both PSTPN and STPN group received more amino acids during the first days of life compared to ITPN due to a change in the local nutrition protocol.

\section{Conclusion}

TPN with a (partially) standardized composition is at least non-inferior to ITPN. The observed differences in weight gain between the groups may be biased by a change in the local nutrition protocol during the study period.

\section{Key notes}

In March 2012, MMC switched to an all-in-one TPN with a partially standardized composition (Numeta ${ }^{\circledR} \mathrm{G} 13 \% \mathrm{E}$ ) and in December 2013 to an all-in-one TPN with a completely standardized composition (NEOmix) consequently.

TPN with a (partially) standardized composition is at least non-inferior to ITPN.

\section{Conflicts of Interest}

None.

\section{Abbreviations}

TPN: total parenteral nutrition; GA: gestational age; MMC: Maxima Medical Center; ITPN: individualized total parenteral nutrition; PSTPN: partially standardized total parenteral nutrition; STPN: standardized total parenteral nutrition; NICU: neonatal intensive care unit; IRB/IEC: Institutional Review Board/Independent Ethics Committee; WMO: Dutch Law on Medical Research with Humans; RDS: respiratory distress syndrome; NEC: necrotizing enterocolitis; IVH: intraventricular hemorrhage; SD: standard deviation

\section{References}

1. Doublet J, Vialet R, Nicaise C, Loundou A, Martin C, Michel F. Achieving parenteral nutrition goals in the critically ill newborns: standardized better than individual- 
ized formulations? Minerva Pediatr. 2013;65(5):497-504.

2. Lafeber HN, Zoeren-Grobben D van, Beek RHT van, Christmann V. Werkboek enterale en parenterale voeding van pasgeborenen. 3rd ed. Amsterdam: VU uitgeverij; 2012.

3. Valentine CJ, Fernandez S, Rogers LK, Gulati P, Hayes J, Lore P, Puthoff T, et al. Early amino-acid administration improves preterm infant weight. J Perinatol. 2009;29(6):428-432.

4. Turpin RS, Canada T, Liu FX, Mercaldi CJ, Pontes-Arruda $A$, Wischmeyer $P$. Nutrition therapy cost analysis in the US: pre-mixed multi-chamber bag vs compounded parenteral nutrition. Appl Health Econ Health Policy. 2011;9(5):281-292.

5. Iacobelli S, Bonsante F, Vintejoux A, Gouyon JB. Standardized parenteral nutrition in preterm infants: early impact on fluid and electrolyte balance. Neonatology. 2010;98(1):84-90.

6. Riskin A, Shiff Y, Shamir R. Parenteral nutrition in neonatology - to standardize or individualize? Isr Med Assoc J. 2006;8(9):641-645.
7. Lafeber HN, Zoeren-Grobben D van, Beek RHT van, Gerards LJ. Werkboek enterale en parenterale voeding van pasgeborenen. 2nd ed. Amsterdam: VU uitgeverij; 2003.

8. Anchieta LM, Xavier CC, Colosimo EA, Souza MF. Weight of preterm newborns during the first twelve weeks of life. Braz J Med Biol Res. 2003;36(6):761-770.

9. Smolkin T, Diab G, Shohat I, Jubran H, Blazer S, Rozen GS, Makhoul IR. Standardized versus individualized parenteral nutrition in very low birth weight infants: a comparative study. Neonatology. 2010;98(2):170-178.

10. Bertino E, Coscia A, Mombro M, Boni L, Rossetti G, Fabris C, et al. Postnatal weight increase and growth velocity of very low birthweight infants. Arch Dis Child Fetal Neonatology. 2006;91(5):349-356.

11. Moyses HE, Johnson MJ, Leaf AA, Cornelius VR. Early parenteral nutrition and growth outcomes in preterm infants: a systematic review and meta-analysis. Am J Clin Nutr. 2013;97(4):816-826.

12. SmPC Numeta G13\%E. Date of first approval 7 April 2011. 